\title{
Las Nuevas Tendencias del Comercio y Negocios Electrónicos en el Sector de Ventas al Detalle para Enfrentar la Crisis Actual
}

\section{New Trends in Electronic Commerce and Business in the Retail Sales Sector to Face the Current Crisis}

\author{
Armando Martín JIMÉNEZ-RODRÍGUEZ1
}

1. Universidad Simón Bolívar, Facultad de Ciencias Sociales y Administrativas. Caracas, Venezuela.

\section{Resumen}

Este artículo parte de una investigación de carácter cualitativo en la que se analizó las principales consecuencias de la covid-19 en la economía mundial y los cambios que la pandemia ha generado en el mercado, en concreto en el comercio y los negocios electrónicos y, especificamente, en el sector de ventas al detalle.

La covid-19 apresuró la urgencia de los actores de la economía global de adecuarse a las ventas en línea. Esta investigación se justifica por la necesidad de conocer el comportamiento del comercio y los negocios electrónicos frente a la pandemia, pues ello permite adecuar a las empresas a esta realidad, para no perder competitividad o verse forzadas a cerrar.

El objetivo fue analizar los principales cambios de la economía mundial con enfoque en las modificaciones que han experimentado el comercio y los negocios electrónicos producto de la pandemia, para generar propuestas de cambio ante la nueva realidad económica.

\section{Palabras clave}

Comercio y negocios electrónicos, ventas al detalle, relaciones económicas internacionales, consumo.

\section{Abstract}

This article is based on a qualitative research that analyzes the main consequences that covid-19 has had for the world economy and the changes that this pandemic has generated in the market, specifically in electronic commerce and business, specifically in the retail sector.

Covid-19 hastened the urgency of global economic players to adjust to online sales. This research justifies the need to know the behavior of commerce and electronic businesses in the face of the pandemic, as this allows companies to adapt to this new reality, since if they do not do so they would lose competitiveness, thus forcing their possible closure.

The aim of this research effort is to analyze the main changes in the world economy, and more focused on the changes that commerce and electronic businesses have had as a result of the pandemic, generating proposals for change in the face of the new economic reality.

\section{Keywords}

E-commerce and business, retail sales, international economic relations, consumption

Códigos de clasificación JEL: F13, L10, L81, M30 


\section{Introducción}

La pandemia causada por la covid-19 ha tenido repercusiones en todos los ámbitos a nivel internacional y la economía mundial no escapó a ello. Los cierres de fronteras nacionales afectaron considerablemente las actividades de distribución, comercialización y producción de los bienes y servicios a escala planetaria.

Dados estos cambios, las empresas se han visto en la necesidad de adecuar su planificación, organización y, en general, su microentorno, para adecuarse a las nuevas realidades comerciales y al crecimiento del comercio electrónico como forma de llegar al consumidor final. Si bien el crecimiento del comercio electrónico no es nuevo, porque data de finales de la década de 1990 del siglo XX y principios del siglo XXI, este ha experimentado un auge por efecto de la pandemia, sobre todo en el sector retail' (Schneider, 2013; López Ruiz, 2018, febrero 7).

Tal vez, uno de los aspectos más relevantes del comercio electrónico en el retail ha sido acostumbrar al consumidor a las compras en línea en productos que este prefería adquirir en los mercados físicos. Alí es donde se ha producido un cambio cultural del consumidor, quien ha modificado sus patrones de consumo (Xu, 2018). Es imposible proyectar si ese cambio permanecerá a futuro una vez finalizada la pandemia, pero lo cierto es que el comercio electrónico continuará creciendo dados los cambios e inversiones en innovación y desarrollo que las empresas han venido aplicando en sus plataformas digitales, además de la seguridad que han adquirido la mayoría de los consumidores respecto a las compras en línea (Betancur Gálvez, 2021).

La investigación es de tipo cualitativo porque se utilizó la recolección y análisis de datos para revelar nuevas interrogantes en el proceso de interpretación. Además, tiene un enfoque mixto para obtener una perspectiva más amplia y profunda del comercio y los negocios internacionales. Cabe mencionar que el objetivo fue analizar los principales cambios de la economía mundial, específicamente las modificaciones que han experimentado el comercio y los negocios electrónicos producto de la pandemia, para proponer algunos cambios que deben aplicar las empresas para adecuarse a esta nueva realidad, con énfasis en la región latinoamericana (Ríos, s.f.; Montes, 2021).

Se considera como aporte de esta investigación no sólo la actualidad del tema, sino también la descripción y análisis de las principales características del comercio electrónico en su sector retail, además de las propuestas de adecuación de las empresas a las nuevas tendencias de las plataformas digitales. Si bien estas últimas no son nuevas, por efecto de la covid-19 han demandado la adecuación tecnológica, operativa y gerencial de las empresas para que puedan continuar con sus operaciones (Cardozo, 2021). Se abordó la región latinoamericana como un todo porque un análisis detallado de por cada país excede el objetivo de esta investigación, conociendo que hay características particulares para cada país, empero, este desarrollo particular genera oportunidades de estudios por caso, los cuales podrían desarrollarse a futuro.

La investigación se dividió en cuatro partes. En la primera se desarrolló el contexto mundial, donde se abordaron los principales efectos de la covid-19 en la economía mundial, con énfasis en las consecuencias en el comercio electrónico y en el papel de la región latinoamericana en relación con esos cambios. En La segunda parte se desarrollaron los aspectos teóricos del comercio y los

1 Cuando se utiliza el término retail (palabra de origen inglés que traducida significa comercio al detalle), se hace referencia a las ventas al consumidor final. Algunos ejemplos de retail son farmacias, ferreterías, supermercados, tiendas de artículos para el hogar, librerías, tiendas de ropa (Moliní Fernández, 2002). 
negocios electrónicos, su importancia y sus características, sobre todo en el subcontinente latinoamericano. El tercer apartado hace referencia a los cambios que se consideran necesarios para adecuar las empresas a esta coyuntura de la economía mundial. Por último, en el cuarto apartado se incorporaron las consideraciones finales a efecto de cumplir con el objetivo general de la investigación.

\section{Efectos de la covid-19 en la economía mundial}

Hoy más que nunca la economía mundial enfrenta cambios importantes que afectan a las actividades de producción, comercialización y distribución de los bienes y servicios a escala planetaria (Gayá, 2015, octubre 20). Las consecuencias de la covid-19 unidas a la digitalización de la economía han influido en el desarrollo de nuevas formas de actuar dentro de una economía cada vez más interconectada a pesar de la pandemia (Cardozo, 2021).

De acuerdo con las Naciones Unidas y la Comisión Económica para América Latina (ONU \& CEPAL, 2020), el inicio de la covid-19 se produjo en una coyuntura de debilitamiento del intercambio de bienes y servicios que se venía produciendo desde la crisis financiera de los años 2008-2009. Así, la propagación acelerada de la covid-19 y las medidas implementadas por los gobiernos han repercutido de forma negativa en las principales economías y, en general, en la macrounidad geográfica mundial. En este contexto, gran parte de las actividades productivas se han interrumpido, primero en Asia y posteriormente en Europa, América del Norte y el resto del mundo, además del cierre generalizado de fronteras (Ríos, s.f.).

Entre las principales consecuencias se puede identificar un marcado aumento del desempleo con la consecuente contracción de la demanda de bienes y servicios. Este aspecto se denota en la mayor reduc- ción del producto global para el año 2020 desde la Segunda Guerra Mundial (ONU \& CEPAL, 2020).

Tal aseveración se desprende de las siguientes estadísticas:

en mayo de 2020 el volumen del comercio mundial de bienes cayó un 17.7 \% con respecto al mismo mes de 2019. La caída en los primeros cinco meses del año fue generalizada, si bien afectó especialmente a las exportaciones de los Estados Unidos, Japón y la Unión Europea. China experimentó una contracción menor que el promedio mundial, ya que controló el brote y reabrió su economía relativamente rápido. América Latina y el Caribe es la región en desarrollo más afectada. En un contexto mundial de mayor regionalización e integración de la producción, la integración regional debe desempeñar un papel clave en las estrategias de salida de la crisis en América Latina y el Caribe. Para avanzar en la integración regional, la infraestructura y la logística deben formar parte de los paquetes de medidas de recuperación económica. Además de su relevante participación directa en el PIB y el empleo, son fundamentales para la producción de todos los bienes y servicios, el suministro de alimentos y servicios esenciales, y la competitividad internacional del comercio. (ONU \& CEPAL, 2020, p. 1)

Unido a esta caída del Producto Interno Bruto (PIB) mundial, paradójicamente, se constata que el intercambio de bienes y servicios mediante las plataformas digitales se ha incrementado vertiginosamente (Montes, 2021). Así, el comercio electrónico en el mundo está pasando por el mejor momento histórico registrado hasta ahora. Tal aseveración se deriva del siguiente planteamiento:

Las proyecciones mundiales a cierre de 2020, señalan al comercio electrónico como el mayor retailer del mundo, según Euromonitor International, con un crecimiento super acelerado debido al alto margen de conexiones de internet en el mundo y a las dinámicas de consumo pre 
y post covid-19. En los últimos años hemos visto cómo ha aumentado la penetración de internet global, creciendo casi 20 puntos entre 2015 y 2020, según Internet World Stats. Hoy tenemos más de 1.500 millones de personas más conectadas a internet de lo que teníamos hace poco más de un lustro. (Castelló Pastor, 2021, p.2)

Por ello, resulta significativo revisar las oportunidades y retos que tiene el subcontinente latinoamericano dentro de esta tendencia que ha adquirido mayor relevancia fruto de la covid-19, y por la necesidad que tienen los actores y agentes económicos de continuar en el mercado y ampliarlo (Schiavini, 2019, marzo 11).

América Latina ha incrementado su participación en internet, pasando de $43.4 \%$ a 71.5 \%, entre los años 2015 y 2020, lo que representa un índice superior al promedio mundial actual que es de $62 \%$, a pesar de encontrarse muy distante en relación con Norteamérica (90.3\%) o Europa (87.2 \%) (Gayá, 2015, octubre 20).

El incremento de la conectividad tanto regional como mundial junto con las cuarentenas y cierres de fronteras está coadyuvando al crecimiento del comercio electrónico y de la industria que lo soporta. Por esta razón es relevante analizar el comportamiento del comercio electrónico, las características del sector retail y los avances logrados por América Latina en esa área; además de las innovaciones que deben hacer las empresas para adaptarse al mercado y continuar creciendo (Moliní Fernández, 2002; Deltoro et al., 2012).

\section{Características y tendencias del comercio y los negocios electrónicos}

La teoría más vinculada al comercio y los negocios electrónicos, de acuerdo con su comportamiento actual, es la teoría de la difusión de la innovación, la cual fue desarrollada por
Rogers (1995). Esta teoría sirve para analizar, desde la perspectiva sociológica, al usuario como elemento esencial en la planificación de los servicios de información (Pérez Pulido \& Terrón Torrado, 2004).

La teoría de la difusión de la innovación de Rogers (1995) ofrece un trabajo conceptual que discute la aceptación de la tecnología a partir de la interrelación de ésta con las dimensiones sociales y psicológicas del usuario, y presenta un análisis teórico preciso que investiga la difusión de la innovación en este sentido. A partir de las definiciones de difusión - proceso por el cual una innovación se comunica por cientos de canales a través del tiempo entre individuos de un sistema social- $y$ de innovación -idea, práctica u objeto percibido como nuevo por un individuo u otra unidad de adopción, Rogers (1995) plantea un modelo teórico basado en cinco elementos - la innovación, los canales de comunicación, el tiempo y el sistema social-identificables en toda investigación sobre difusión, y un proceso de decisión de la innovación dividido en varias etapas, que el individuo o la organización han de superar para alcanzar el definitivo grado de adopción de una innovación. (Pérez Pulido \& Terrón Torrado, 2004, p. 309)

Analizando los términos mencionados, se puede acotar que, de acuerdo con el planteamiento de Rogers (1995), existen varias etapas relevantes de esta teoría, las cuales se explicarán a continuación.

La primera de ellas se refiere al conocimiento, entendido como el periodo en el que el usuario es receptivo a la innovación. En esta etapa es fundamental comunicar sobre la innovación al consumidor para que asimile y tome decisiones respecto a la institución u organización. La segunda etapa consiste en la actitud de la formación, es decir, el usuario determina la utilidad de la innovación para aplicarla. La etapa tercera corresponde a la toma de decisiones, la cual se refiere al convencimiento de los agentes de cambio para cumplir con la siguiente etapa. La cuarta consiste en 
aplicar esa innovación para, posteriormente, pasar a la última etapa, en la cual se confirma el uso de la innovación, que puede ser continuo o discontinuo (permanecer en el tiempo o no) (Schneider, 2013; Andrade Muñoz, s.f.). Volviendo a Rogers (1995), la innovación debe tener los siguientes atributos:

1. Presentar ventajas, es decir, tener aspectos positivos que justifiquen su aplicación;

2. Ser sencilla, entendible y aplicable, lo que facilitará su puesta en marcha;

3. Convivir con sistemas ya existentes para facilitar su aplicabilidad.

4. Poder ser probada, es decir, que se pueda experimentar con ella.

5. Ser medible, es decir, poder medir sus resultados (Xu, 2018; Marketing Digital, 2015, agosto 12).

Las innovaciones pueden ser radicales y complementarias. Las primeras son las que rompen con tecnologías existentes y generan nuevas tecnologías, por ejemplo, el salto tecnológico de VHS y Betamax a Compact Disc (CD). Las segundas corresponden a tecnologías que actualizan o conviven con tecnologías anteriores porque las complementan, por ejemplo, el Roku, un adaptador que permite que los televisores de versiones anteriores puedan tener acceso a canales digitales a través del uso del internet, sin necesidad de comprar un televisor adaptado a las nuevas tecnologías (Betancur Gálvez, s.f.).

Antes de abordar la importancia del comercio electrónico, se presentan sus características, particularidades y definiciones.

El comercio electrónico involucra la utilización de aplicaciones móviles, buscadores, el uso de internet y la World Wide Web para hacer transacciones electrónicas o de negocios (Robayo-Botiva, 2020). En palabras de Robayo-Botiva:

el comercio electrónico describe el proceso de compra, venta, transferencia, servicio o intercambio de productos y servicios o información mediante una red de computadores, incluyendo internet.... Finalmente,... para muchas personas el comercio electrónico significa comprar a través de internet mediante la web. Sin embargo, el comercio electrónico incluye otras actividades, tales como comercio entre negocios y procesos internos que las organizaciones usan para soportar la compra, venta, contratación, planeación y otras actividades. (RobayoBotiva, 2020, p. 5)

En síntesis, se puede decir que el comercio electrónico es el intercambio de bienes y servicios por medio de plataformas digitales, las cuales sirven de instrumento de compra y venta en línea (López Ruiz, 2018, febrero 7).

Los términos negocio y comercio electrónicos no significan lo mismo, aunque a veces se utilicen como sinónimos. A este respecto se establecerán, a partir de Robayo-Botiva (2020) las diferencias:

Negocio electrónico se refiere a las transacciones y procesos habilitados digitalmente dentro de una organización, involucrando los sistemas de información bajo el control de las organizaciones. Adicionalmente, el negocio electrónico no incluye transacciones comerciales que involucren un intercambio de valor a través de los límites de la organización. Por ejemplo, el mecanismo de control de inventario en línea de una organización es un componente del negocio electrónico, pero tales procesos internos no generan directamente ingresos a la organización.... De acuerdo con esto, el comercio electrónico está constituido por transacciones comerciales que se realizan a través de internet, mientras que el negocio electrónico utiliza las tecnologías de información y los sistemas de información, procurando mejorar procesos y transacciones dentro de las organizaciones. Sin embargo, cabe destacar que tanto para el comercio como para el negocio electrónico la infraestructura tecnológica de una organización es fundamental. (p. 6) 
Como se puede ver, el elemento común de ambos términos son las plataformas tecnológicas (Cook \& Sellers, 1997).

Otra de las principales consecuencias de la covid-19 en el comercio electrónico, sobre todo en el sector retail, es el desplazamiento de los consumidores de las compras presenciales tradicionales hacia las compras vía plataformas de comercio electrónico. En consecuencia, todos los sectores y mercados que estaban retrasados tanto en el desarrollo de sus plataformas para compras en línea como en sus sistemas de información se han visto forzados a acelerar su adecuación digital, para no perder mercados $y$ adecuarse a las nuevas realidades del consumidor mundial (Tecnología para los Negocios, s.f.).
Así, es relevante para esta investigación referirse a las tendencias más actualizadas del comercio y negocios electrónicos, que señala Martínez (2021a, abril 15), ya que este año exigirá a las empresas seguir manteniendo un ritmo acelerado de desarrollo y maduración digital si desean continuar en el mercado. Conviene analizar, primero, cuáles son las características principales y actuales del comercio y los negocios electrónicos antes de proponer los principales cambios a los cuales se enfrentan las empresas en esta coyuntura económica mundial (Paz Lloveras, 1998; Castell, 2016).

A tal efecto, se analizan las siguientes estadísticas (figura 1) que presentan un panorama del comportamiento del comercio y los negocios electrónicos para diagnosticar sus principales características.

Figura 1. Porcentaje de crecimiento del comercio electrónico en América Latina

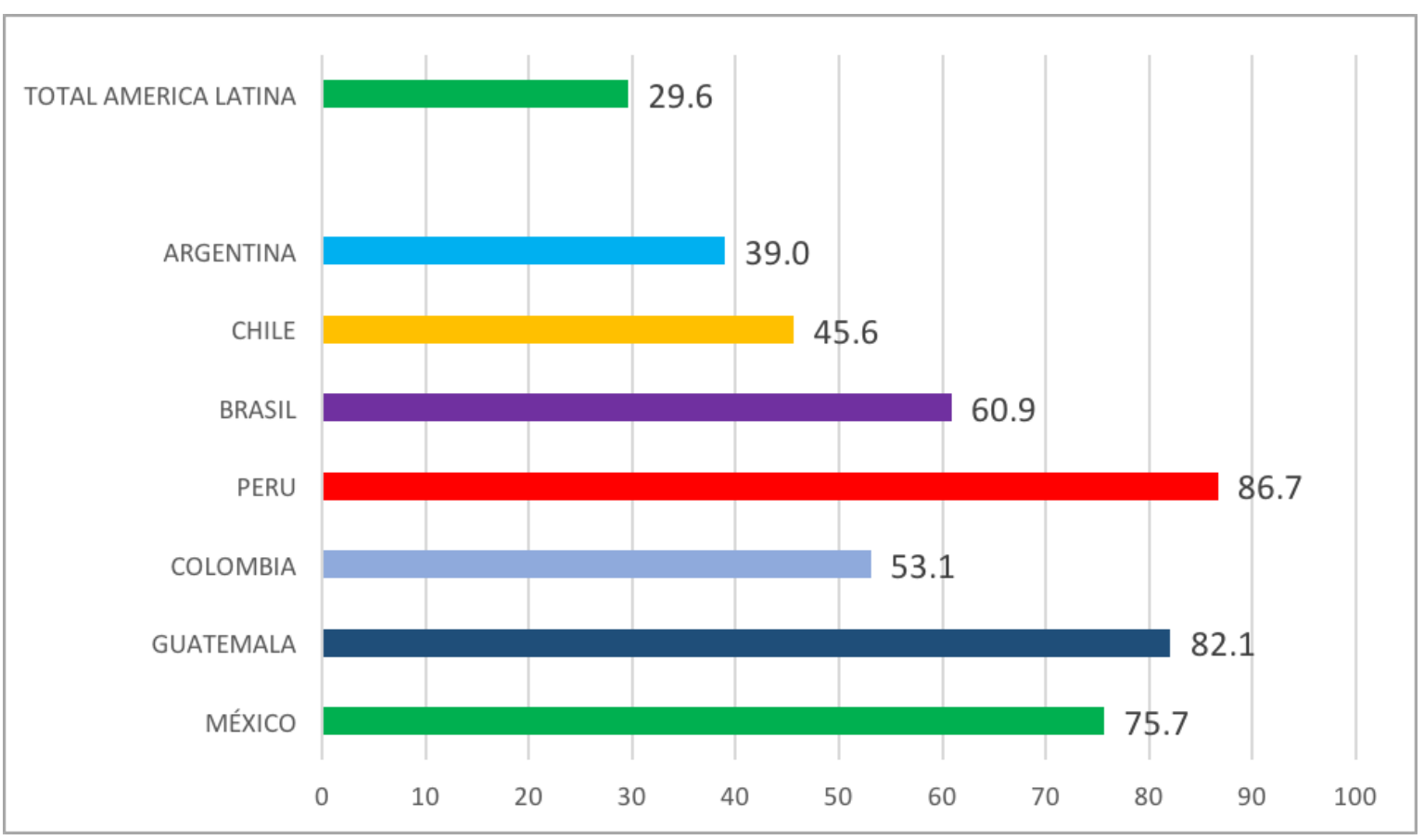

Nota: se excluyen del análisis los productos generados a través de sitios de ventas consumidor a consumidor, ventas de vehículos de motor, motocicletas y piezas de vehículos, entradas para eventos, ventas de paquetes de viajes y vacaciones, e ingresos generados por juegos de azar en línea. Adaptado de los datos de Euromonitor Internacional (2021, enero 19).

En la figura 1, América Latina creció en promedio un $29.6 \%$. El mayor crecimien- to porcentual lo obtuvo Perú, con $86.7 \%$ y el crecimiento de Guatemala alcanza el 
$82.1 \%)$, por lo tanto, este país es el segundo con mayor crecimiento de la región. También se puede constatar crecimientos superiores al promedio de la región en México (75.7\%), Brasil (60.9\%), Colombia (53.1\%), Chile (45.6 \%) y Argentina (39 \%) (Euromonitor Internacional, 2021, enero 19). Lo interesante de estas cifras es que antes de la pandemia existía un aspecto cultural en la región latinoamericana que vinculaba la confianza del consumidor a las compras presenciales. Esa barrera cultural se desvanecido por las restricciones de movilidad contra la covid-19.

Una de las grandes interrogantes es saber si esta tendencia se mantendrá una vez que haya finalizado la pandemia. A este respecto, Montes (2021, marzo 29) aclara que todo dependerá de si las empresas de retail continúan invirtiendo y apostando por el comercio electrónico, evitan las demoras en las entregas y potencian el buen manejo y planificación de los stocks de productos. Esta apreciación es conveniente, pero un análisis desde el punto de vista del consumidor obligaría a estudiar si el consumidor final ha asimilado, dentro de sus características psicosociales de compra, la confianza necesaria para adquirir bienes y servicios por medio del comercio electrónico o, por el contrario, esta tendencia responde solamente a los efectos de la covid-19 (Cohen \& Asin, 2000; Conexión ESAN, 2016, octubre 3).

Un aspecto fundamental para esta investigación fue analizar el comportamiento de las compras, específicamente en el sector retail, por la importancia que han adquirido en los volúmenes de ventas a nivel mundial y por la cantidad de sectores que participan en el comercio y los negocios electrónicos. A este respecto, se considera oportuno profundizar en las tendencias de este tipo de comercio.

El Statista Research Department (2015, diciembre 15) proyectó que, para el año 2020, existen más de 2000 millones de consumidores que comprarán bienes y servicios mediante las plataformas de internet y que de ellas un número superior a $267 \mathrm{mi}-$ llones tendrían su origen en Latinoamérica, es decir, aproximadamente un $13.35 \%$ de las compras mundiales del sector retail provendría de la región. Para el año 2024, se proyecta que esta cifra aumentará en un $31 \%$, lo que corresponde a cerca de 351 millones de usuarios en toda la región. Esta cifra puede ser sumamente relevante porque en solo cinco años Latinoamérica pasó de 126 millones de compradores a 267 millones, es decir, hubo un crecimiento del $111.90 \%$.

En las figuras 2 y 3 , donde se proyectan los datos sobre ventas del sector retail por regiones, la región que muestra el mayor crecimiento es Asia-Pacífico, con más del $62 \%$ a nivel mundial; luego le siguen Norteamérica y Europa Occidental. Asimismo, existe un significativo crecimiento del número de consumidores digitales en los últimos años en todo el mundo, donde el sector del retail en línea cuenta cada vez más con cifras de crecimiento.

Latinoamérica no escapa a esta tendencia, pues las ventas en retail en línea pasaron de 36900 USD millones en 2016 a 83630 millones USD en 2020, casi triplicando de esta manera su valor en menos de cinco años. La predicción para 2023 es de 116230 millones. Entre los factores que se considera que han favorecido a esta tendencia alcista no solo figuran los efectos de la covid-19, sino también el incremento de aplicaciones para compras en línea desde las computadoras, junto con las de teléfonos celulares y tablets, las cuales han facilitado el uso para las compras en línea. 
Figura 2. Ventas de retail por comercio electrónico, año 2020

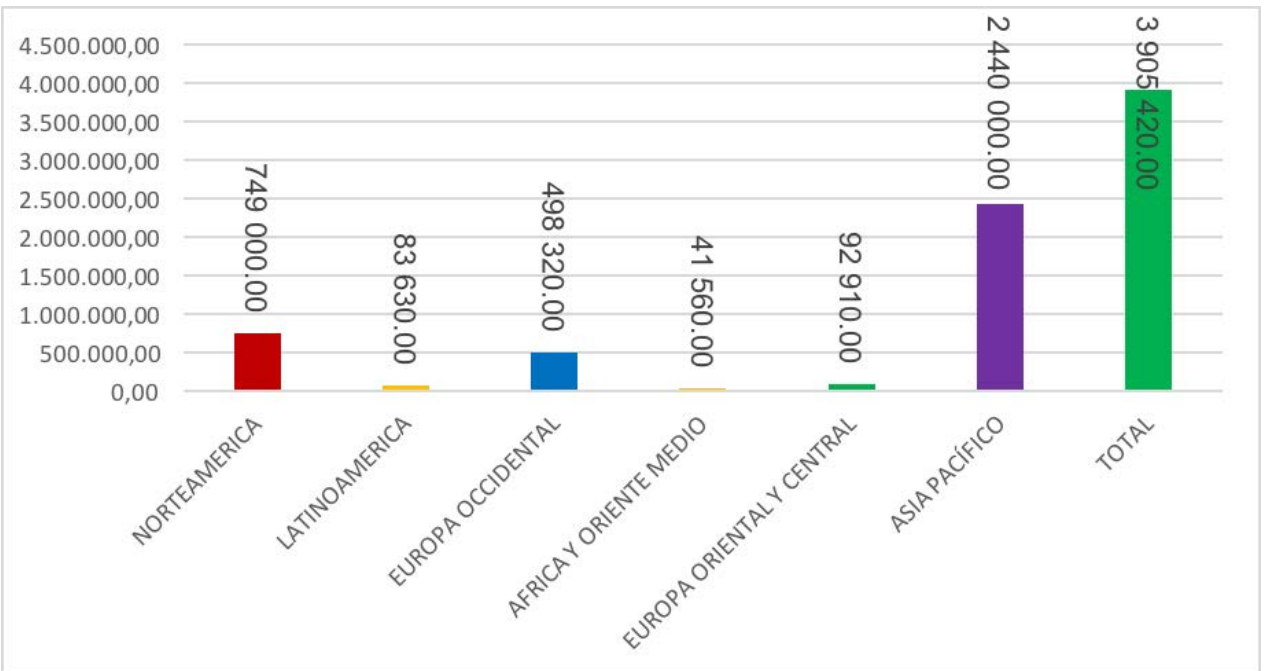

Nota: Adaptado de la proyección de datos de Statista Research Department (2015, diciembre 15).

Figura 3. Participación porcentual de ventas del sector retail por región geográfica, año 2020

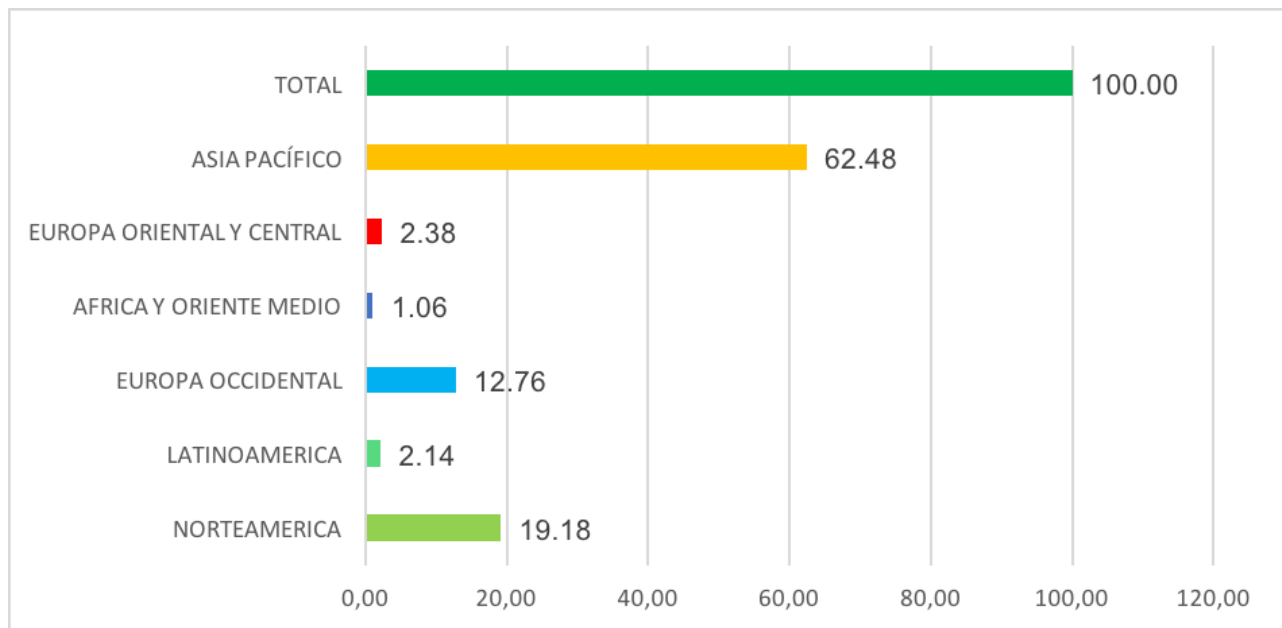

Nota: Adaptado de la proyección de datos de Statista Research Department (2015).

\section{Estrategias empresariales para la adecuación del comercio y los negocios electrónicos frente a las consecuencias de la covid-19}

Una vez mencionadas y analizadas las cifras relevantes sobre el comercio y los negocios electrónicos y la importancia del sector retail en este ámbito, se expondrá cuáles se consideran que deben ser las estrategias empresariales para enfrentar las consecuencias del covid-19.

\section{Cambios en medio de las nuevas realidades del comercio y los negocios electrónicos}

En un momento de tanta incertidumbre es fundamental la planificación, organización, 
comunicación y capacidad de respuesta de los elementos sustanciales para tomar decisiones empresariales (Polastri, 2020, agosto 13). A continuación, se presentan los principales elementos que han surgido del análisis y que se consideran sustanciales para res- ponder a esta coyuntura (Rubio, 2014, mayo 14). En la figura 4 constan los principales cambios que enfrentan las empresas en medio de las nuevas realidades del comercio y los negocios electrónicos:

Figura 4. Principales procesos de adaptación de las empresas al comercio y negocios electrónicos del sector retail como consecuencia de la covid-19

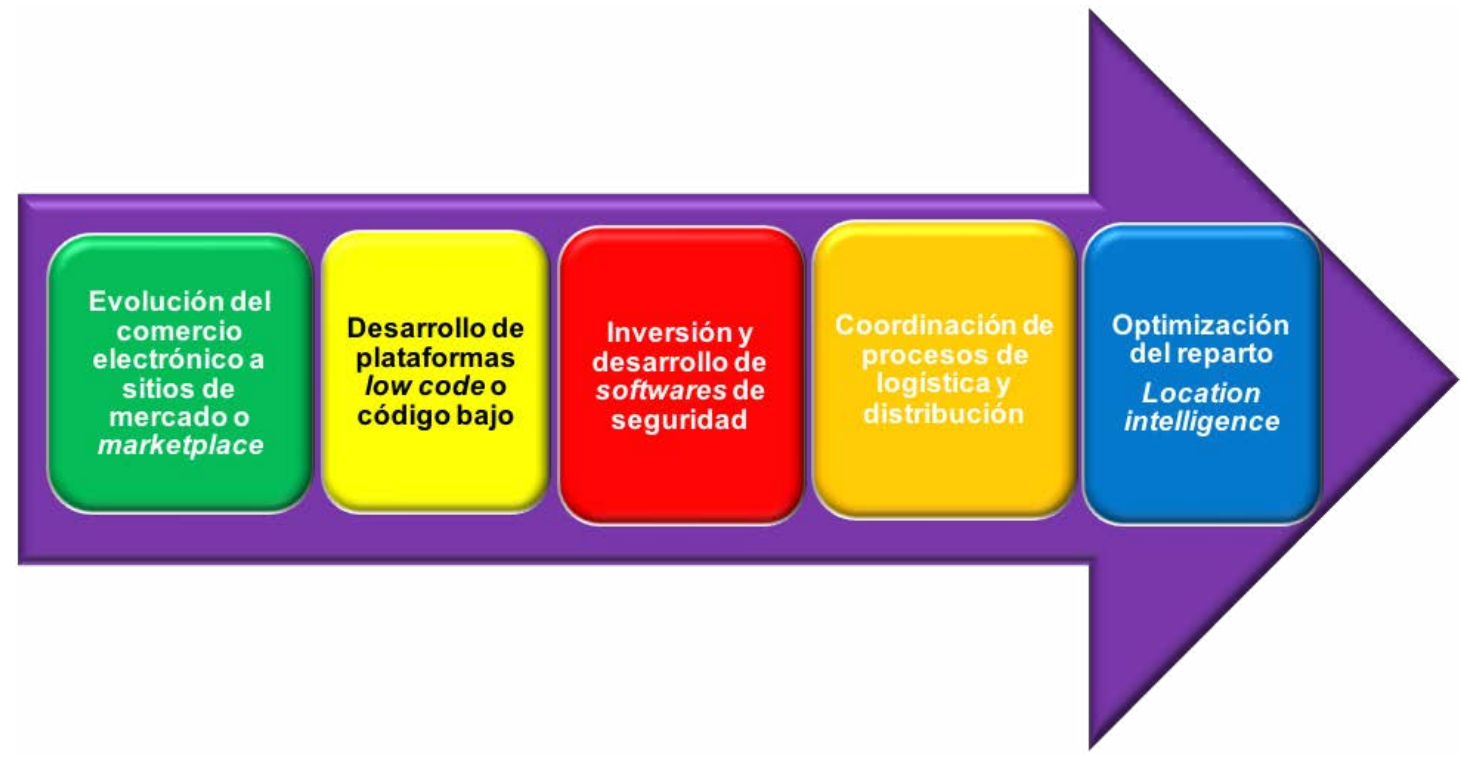

\section{Evolución del comercio electrónico a sitios de mercado o marketplace}

Un marketplace o lugar de mercado puede ser una aplicación o sitio web donde se encuentran variedades de productos y personal que atiende las necesidades del consumidor en la misma plataforma o aplicación. Los ejemplos más conocidos son Mercado Libre, Amazon, entre otros. Martínez (2021b, mayo, 10, p.1) y De la Rica (1997) explican: "este tipo de plataformas son efectivas para diversificar la oferta del e-commerce y mejorar su rentabilidad... el $41 \%$ de las ventas online suceden en un marketplace".

La evolución del negocio electrónico reside en desarrollar un centro de ventas o seller center que consiste en tener una pla- taforma digital que permita organizar y mantener una operación diaria que cumpla con cuatro funciones principales:

1. Controlar el inventario de productos.

2. Reunir a todos los compradores y vendedores en un solo lugar.

3. Administrar las estrategias de promoción y ventas

4. Permitir que los compradores puedan controlar y administrar sus operaciones en un solo lugar.

Esto coadyuva a las empresas a adecuarse a las características del consumidor, quien no desea revisar un sinfín de plataformas digitales para hacer sus compras si no tiene una atención adecuada. 


\section{Desarrollo de plataformas low code o código bajo}

Otro aspecto relevante sobre los cambios más significativos a los cuales se enfrentan las empresas dentro de esta coyuntura es el desarrollo de plataformas low code o código bajo. La forma como se están construyendo aplicaciones también ha experimentado modificaciones importantes, debido a que se trata de un método de programación que requiere poca programación manual, lo que implica que no hay que tener grandes conocimientos informáticos para desarrollarlas.

Su objetivo principal radica en la accesibilidad para todo el mundo, incluso para profesionales que no sean desarrolladores expertos. Estas aplicaciones brindan varias ventajas que contribuirían a las empresas en su incursión al comercio electrónico y han presentado hasta ahora estadísticas relevantes. De acuerdo con Cera (2021, mayo 17), para el año 2021, se estima un crecimiento del $23 \%$ en el desarrollo de plataformas mediante low code y para el 2024, un $65 \%$ del desarrollo producto de esta tendencia.

Analizando este cambio al cual se deben adecuar las empresas, conviene enumerar las ventajas del incremento de este tipo de desarrollo low code. Sus principales ventajas son:

a. Reducción de costos: se dedica menos tiempo para su desarrollo, con lo cual se garantiza agilidad en su aplicación y las empresas tienen mayor capacidad de adaptación a las nuevas tendencias del comercio electrónico. Algunos ejemplos son Xplenty, Outsystem, Appian, Salesforce Lightning, entre otras.

b. Mejora de la productividad: por ser un desarrollo más simple, se reduce el tiempo y mejora la competitividad de las empresas. Se estima que se reduce el tiempo en el desarrollo de aplicaciones en un $90 \%$. c. Desarrollo mucho más rápido: se ahorra tiempo para conseguir que una determinada aplicación funcione y satisfaga una necesidad. Es una forma rápida de desarrollar una aplicación y de adaptarse a las necesidades del consumidor y de la empresa. Consecuentemente, las compañías desarrollan ventajas competitivas frente a sus competidores, porque se adaptan rápidamente a las necesidades del consumidor. Las ventajas competitivas, en este caso, se refieren a la capacidad que deben tener los actores económicos para desarrollar e invertir en innovaciones y tecnologías que les permitan, por un lado, adaptarse a las necesidades del consumidor $y$, por otro, ampliar sus mercados frente a los competidores.

\section{Inversión y desarrollo de softwares de seguridad}

La necesidad de inversión y desarrollo de softwares de seguridad para que los clientes tengan confianza al momento de comprar en línea: La pandemia del covid-19 forzó a que muchos consumidores confíen en las compras en línea, por lo que es fundamental, para ampliar el mercado, que los sistemas de seguridad de compras en línea sean lo suficientemente seguros y confiables para que el usuario pueda, sin temor alguno, acceder a la plataforma de compras; de lo contrario el cliente no comprará.

\section{Coordinación de procesos de logística y distribución}

La importancia de la coordinación de procesos de logística y distribución: los procesos de logística y distribución están vinculados al comercio y los negocios electrónicos porque deben responder acorde a las exigencias y demandas de los usuarios y no 
provocar una ralentización en la industria, debido a que la entrega inmediata es una característica esencial que exige el consumidor. Si bien por la pandemia del covid-19 se han cerrado fronteras y se han afectado las actividades del transporte internacional, el dropshipping es una estrategia que juega un papel fundamental en este aspecto, porque las empresas en línea ya no necesitan un almacén para guardar los productos, sino que lo pueden adquirir de una tercera persona. De esta forma la empresa puede reducir los gastos derivados de la gestión de stock.

\section{Optimización del reparto o location intelligence}

Actualmente la optimización del reparto es esencial. La distribución se ha venido perfeccionando mediante el desarrollo de las tecnologías denominadas location intelligence. Este tipo de tecnologías utilizan datos geolocalizados en tiempo real y permiten definir cuál es el mejor camino. Incluso pueden coadyuvar a rectificar las rutas instantáneamente si se ha producido un cambio resaltable en las ellas.

\section{Estrategias empresariales}

Para aplicar estos principales procesos exitosamente se deben hacer los cambios que se propusieron anteriormente. La adaptación de las empresas a los canales digitales debe ser un hecho. Desde hace aproximadamente 10 años contar con una página web y un catálogo de productos en línea es insuficiente (ESNECA Business School, 2020, junio 16). Es prioritario que las empresas comiencen a invertir en innovación y desarrollo de aplicaciones, no solo en computadoras sino también para tablets y celulares, que le permitan al consumidor final conocer las características de sus productos y servicios, además de una plataforma de pagos confiable para la psiquis del cliente. Por esta razón, el desarrollo del marketplace es fundamental si las empresas desean continuar creciendo, ser competitivas y aumentar sus clientes.

Otro aspecto que se considera esencial es manejar las redes sociales y, a través de estas, acercarse al consumidor. Se considera que las redes sociales no deben ser solo instrumentos de promoción, sino también de acercamiento al consumidor, donde este se sienta identificado con la empresa. En las redes sociales se puede publicar, por ejemplo, aspectos informativos de la pandemia, temas que le sean útiles al momento de consumir, celebración de eventos en línea con contenidos informativos de actualidad, entre otros. Estas acciones generan vínculos con el consumidor, que luego se pueden traducir en fidelidad de marca o productos que son y serán muy útiles para las compañías.

Desde el punto de vista del microentorno empresarial, es básico tener una buena comunicación con los empleados y proveedores. Una comunicación positiva con el capital humano de la empresa y con los proveedores permite tener los objetivos y respuestas claras, además que coadyuva en la planificación estratégica de la empresa y en el manejo del inventario. Este último elemento es esencial para el comercio electrónico.

Por otra parte, además, se considera necesario revisar los objetivos de la empresa. El mundo y específicamente el sector empresarial no está adecuado a la coyuntura actual, por lo que revisar los objetivos de ventas, la planificación de la producción, la inversión en innovación y desarrollo, entre otros elementos esenciales en estos tiempos se considera una reinvención. Es aquí donde el desarrollo de plataformas mediante low code puede jugar un papel fundamental.

Un último aspecto debe ser la gestión de riesgos y manejo de escenarios. El manejo de escenarios de crisis, bien sea por situaciones nuevas que puedan presentarse o por las situaciones actuales que ya se han de- 
sarrollado -cierre de fronteras, problemas con la adquisición de materias primas, entre otros-, es básico para no desviarse de los objetivos y para tener la capacidad de respuesta necesaria para no afectar las operaciones de las compañías.

En sintesis, se puede decir que la pandemia del covid-19 ha generado retos y oportunidades a todas las empresas a nivel mundial. El comercio electrónico ha tenido un incremento importante como canal de ventas a escala planetaria y los actores y agentes económicos se deben adecuar a este si no desean desaparecer del mercado. Si bien el comercio electrónico ya tenía un comportamiento alcista antes de la pandemia, este apresuró la decisión de las empresas de ajustarse ante la crisis y de insertar sus productos y servicios en plataformas digitales que les permita colocar sus productos y servicios en un mercado digital cada vez más creciente y cambiante.

\section{Conclusiones}

Hoy más que nunca la economía mundial enfrenta cambios importantes que afectan las actividades de producción, comercialización y distribución de los bienes y servicios a escala global. Las consecuencias de la covid-19, junto con el acelerado desarrollo de las $\mathrm{TIC}$, han repercutido en nuevas formas de actuar dentro de una economía cada vez más interconectada a pesar de la pandemia.

De acuerdo con ONU y CEPAL (2020), el inicio de la covid-19 se produjo en una coyuntura de debilitamiento del intercambio de bienes y servicios que viene desde la crisis financiera de los años 2008-2009. Así, la propagación acelerada de la covid-19 y las medidas implementadas por los gobiernos han tenido un impacto negativo en las principales economías y, en general, en la macrounidad geográfica mundial.

Entre las principales consecuencias se puede destacar un marcado aumento del desempleo, con la consecuente contracción de la demanda de bienes y servicios, lo cual se puede corroborar en la mayor reducción del producto global para el año 2020 desde la Segunda Guerra Mundial (ONU \& CEPAL, 2020). La covid-19 ha causado el desplazamiento de los consumidores de las compras presenciales tradicionales hacia las compras vía plataformas de comercio electrónico. Esto ha traído como consecuencia que todos los sectores y mercados que estaban retrasados en el desarrollo de sus plataformas para compras en línea se vean forzados a acelerar su adecuación digital, para no perder mercados y adaptarse a las nuevas realidades del consumidor mundial. Así, es relevante puntualizar que el contexto actual exigirá a las empresas seguir manteniendo un ritmo acelerado de desarrollo y maduración digital si desean continuar en el mercado bien sea nacional o internacional.

La pandemia de la covid-19 ha generado retos y oportunidades a todas las empresas a nivel mundial. El comercio electrónico ha experimentado un incremento importante como canal de ventas a escala planetaria y los actores y agentes económicos se deben adecuar a este si no desean desaparecer del mercado. Si bien el comercio electrónico ya tenía un comportamiento alcista antes de la pandemia, esta apresuró la decisión de las empresas de ajustarse ante la crisis e insertar sus productos y servicios en plataformas digitales en un mercado digital cada vez más creciente, cambiante y competitivo.

\section{Referencias}

Andrade Muñoz, J. (s.f.). Comercio electrónico. https://bit.ly/3EtC1B9

Betancur Gálvez, C. (s.f.). Comercio electrónico: Cómo ha cambiado con el paso del tiempo. BTODigital. Consultado en junio 27, 2021, de https://bit.ly/3Bfrl6W

Cardozo, N. D. (2021). La acción pública en el marco de la pandemia del COVID-19. Algunas 
reflexiones desde América Latina. En El COVID-19: Crisis, desafíos y nuevas estrategias socioeconómicas y culturales (M. F. Gutiérrez \& M. M. Llairó, Comps., pp.115128). Imago Mundi.

Castell, A. C. (2016). Reducing order-to-delivery time using collaborative supply chains in global industries. [Tesis doctoral, Northcentral University]. ProQuest Dissertations Publishing. https://bit.ly/3BcffLG

Castelló Pastor, J.J. (2021). Desafíos jurídicos ante la integración digital: aspectos europeos e internacionales. Aranzadi.

Cera, C. (2021, mayo 17). Low-code: ¿Desarrollo fácil o el futuro de la programación? Appvizer. https://bit.ly/2ZfHdZR

Cohen, D., \& Asin, E. (2000). Sistemas de información para los negocios: Un enfoque de toma de decisiones (3. ${ }^{a}$ Ed.). McGraw-Hill.

Conexión ESAN. (2016, octubre 3). Indicadores de medición en el comercio electrónico. Conexión ESAN. https://bit.ly/30Mh8Cn

Cook, D., \& Sellers, D. (1997). Inicie su negocio en web. Prentice-Hall Hispanoamericana.

De la Rica, E. (1997). Marketing en internet. ESIC.

Deltoro, M., Descals, A., \& Molina, M. (2012). Factores determinantes y consecuencias de la adopción del comercio electrónico B2C: Una comparativa internacional. Estudios Gerenciales, 28(123), 101-120. https://bit. ly/2XHiOgN

ESNECA Business School. (2020, junio 16). ¿Cuáles son las ventajas de los negocios electrónicos? https://bit.ly/3Cbt2DD

Euromonitor International. (2021, enero 19). Las 10 principales tendencias globales de consumo para 2021. https://bit.ly/3jD0jk7

Gayá, R. (2015, octubre 20). El comercio electrónico y la inserción internacional de América Latina y el Caribe. Banco Interamericano de Desarrollo. https://bit.ly/3B9o5dq

López Ruiz, A. (2018, febrero 7). Comercio electrónico: Definición, tipos y tendencias. Rankia. https://bit.ly/3EhLKtW

Marketing Digital. (2015, agosto 12). ¿Qué es e-commerce o comercio electrónico? Marketing Digital: Blog del Máster en Marketing Directo y Digital de la UPF Barce-
Iona School of Management. https://bit. ly/3Cfv9Xk

Martínez, C. (2021a, abril 15). Las cifras clave del eCommerce en el mundo en 2021. BlackSip. Consultado en abril 15, 2021, de https://bit.ly/3vJdrsB

Martínez, C. (2021b, mayo 10). Los datos y cifras clave del eCommerce en Latinoamérica 2021. BlackSip. Consultado en mayo 10, 2021, de https://bit.ly/3Gui7rs

Moliní Fernández, F. (2002). Ventajas, inconvenientes e impactos territoriales del comercio electrónico. Investigaciones Geográficas, (27), 131-150. https://bit.ly/3vJfpJv

Montes, S. (2021, marzo 29). El comercio electrónico en la región creció 66 \% en 2020 y llegó a US\$66.765 millones. LR (La República). https://bit.ly/3jAjWJA

Organización de las Naciones Unidas (ONU) \& Comisión Económica para América Latina y el Caribe (CEPAL). (2020). Los efectos del COVID-19 en el comercio internacional y la logística. CEPAL.

Paz Lloveras, E. (1998). Cómo exportar, importar y hacer negocios a través de internet: Guía profesional para la gestión de la exportación, importación y cooperación empresarial a través de internet. Gestión 2000.

Pérez Pulido, M., \& Terrón Torrado, M. (2004). La teoría de la difusión de la innovación y su aplicación al estudio de la adopción de recursos electrónicos por los investigadores en la Universidad de Extremadura. Revista Española de Documentación Científica, 27(3), 308-329. https://doi.org/10.3989/ redc.2004.v27.i3.155

Polastri, G. (2020, agosto 13). Las claves para digitalizar tu negocio, por Gianfranco Polastri. El Comercio. https://bit.ly/3mesUxS

Ríos, M. D. (s.f.). El pequeño empresario en ALC, las TIC y el comercio electrónico. Monografías.com. Consultado en agosto 10, 2021, de https://bit.ly/3GleyUg

Robayo-Botiva, D. (2020). El comercio electrónico: Concepto, características e importancia en las organizaciones. Revista Generación de Contenidos Impresos, (20). doi: https://doi.org/10.16925/gclc.13 
Rogers, E. (1995). Difussion of Innovation (4. ${ }^{\mathrm{a}}$ Ed). The Free Press.

Rubio, J. M. (2014, mayo 15). Tiendas online para mayoristas y fabricantes. Sugerendo: Engineering Commerce. https://bit. ly/3bdOTAd

Schiavini, R. (2019, marzo 11). El futuro del comercio electrónico: 10 tendencias para los próximos años. SmartHint Blog. https:// bit.ly/3jCSRFw

Schneider, P. (2013). Comercio electrónico (10. ${ }^{\text {a }}$ Ed.). Cengage Learning.
Statista Research Department. (2015, diciembre 15). Ingresos del comercio minorista móvil a nivel mundial de 2012 a 2018. Statista Research Department. https://bit.ly/3B9k1tC

Tecnología para los Negocios. (s.f.). Tendencias del comercio electrónico: ¿Hacia dónde vamos? Consultado en noviembre 3, 2021, de https://bit.ly/3Bfrl6W

Xu, Z. (2018). Essays on Online Retailing in Emerging Markets. [Tesis doctoral, Northwestern University]. ProQuest Dissertations Publishing. https://bit.ly/3Gm9tu0 